\title{
Analysis on Self-presentation in Internet Social Media
}

\author{
Wei Huang \\ Economics and Management School of Yunnan Normal University, China
}

Keywords: Internet; social media; Goffman; self-presentation

\begin{abstract}
Self-representation on the Internet in social media is totally different from traditional methods in the presentation objects, presentation methods and presentation effects with distinct characteristics of the times and profound impact on individuals and society. However, it is still the true social and real life that plays an important role in online social media. The distinction of social foundations and operational mechanisms behind different media helps to guide people to achieve effective "positive presentation" through institutional construction.
\end{abstract}

\section{Introduction}

Since the beginning of the 21st century, with the rise and development of the Internet, many social media have emerged, QQ, Renren, Microblog, WeChat, Momo and other media. Compared with traditional ways of communication, online social media has distinctive features, which also makes the people's self-presentation ways sharply different from those before.

\section{Distinctive Features from Traditions in Self-presentation in Online Social Media}

\subsection{Self-presentation in online social media with a wide range of presentation objects}

One of the distinguishing features of the Internet is that it breaks through geographical restrictions, which is particularly evident in social media. The original social media is also based on certain area, identity or hobby. In other words, it is based on a specific purpose of communication. The form and nature of the platform make the interaction between strangers become simple and feasible. And the people's self-presentation has also undergone breakthrough changes.

\subsection{Self-presentation in online social media with significant features of selective presentation}

The network has a very powerful feature of selective presentation. Since some platforms, such as Microblog and WeChat, the users can publish the content what they want which is closely related to the image of "self-presentation". The publishers will be very willing to choose those that can shape their own good image and information and completely hide those unfavorable.

\subsection{Self-presentation in online social media is more deceptive with lower costs}

In social networks, people complete their self-presentation through texts and pictures, which only requires the Internet. Self-representation in social networks is quite deceptive because of its low cost. A person can disguise an affluent identity through some false pictures. Similarly, it is easy for a person to disguise his sense of justice and sympathy through cheaper ways such as writing and forwarding comments. Because of this, over time, the network has begun to appear the "slobber party" and "keyboard men", condemning the cheap and costless sense of false morality.

\section{The "Self-presentation in Online Social Media" Constrained by Social Development and Specific Operating Mechanisms}

In order to complete understanding of self-presentation in online social media, we should clearly recognize that although the network is virtual, the images that people present through the network also have certain characteristics of virtuality, incompleteness and concealment. It is the various 
systems that plays an important role in online social media. Based on institutional analysis, it can help us to understand this phenomenon more objectively and completely. It also helps us to guide social development through institutional adjustment.

\subsection{Self-presentation in social media is constrained by the development of the real society}

In a broad sense, what people present through a mask is necessarily certain social and cultural mentality. For example, the Chinese people generally have the mentality of "Guangzong Yaozu and Yijin Huanxiang". However, each kind of "presentation" superficially displays the differenced of culture and society. The role played behind it is the degree of development of the real society. The same is true of self-presentation in online social media. The content of people's "presentation" still stems from the reality of social life and still conforms to the basic principle of Marx's principle of "economic foundation determines the superstructure".

\subsection{Significantly different self-presentation due to different media operating mechanisms}

The self-presentation method under the social media network has distinct styles and characteristics. Under different media operating mechanisms, people's "network self-presentation methods" have significant differences. This article takes Sina Microblog, WeChat and Momo as examples to explore this difference.

Self-presentation under Microblog operation mechanism. The operating mechanism of Microblog has three distinctive features. First, it implements operation mode of "VIP customer + common fans". Second, it has the feature of semi-open nature. Each person in their own Microblog can published their information to unspecified people. Third, the "real-name system" and "anonymity" in Microblog are concurrent. Influential people can apply for real-name authentication while the rest of the general public are anonymous. In order to attract more people, the Microblog platform pays special attention and focuses on recommending some "celebrities" to guide social topics through the celebrity effect. The functions of review and forwarding of Microblog can produce fermentation effects in specific groups of people. Therefore, under the Microblog operation mechanism, it is easy to generate so-called "big V", "web celebrity" and opinion leaders. Microblog's function of "anonymity" has given people certain rights to speak and choose. Therefore, it is easy to form a sensational topic on Microblog. Based on the above features, to certain degree, Microblog is more like a virtual projection of a real society on the Internet, which is equivocal on the surface but has invisible wealth and status differences. All of these has caused that people's "self-presentation" is polarized. The real-name authenticated users tend to guide topics and are willing to "present" their beautifying images while the anonymous users tend to focus on and forward "negative information".

Self-expression under the operation mechanism of WeChat. The operation mechanism of WeChat mainly has two notable features. First, WeChat implements a semi-transparent "human social" model. The "friends" of the WeChat are mainly generated through the phone contacts and QQ friends. Therefore, although it is a network account, there is always a real and specific person behind the WeChat account. And between WeChat friends, they often have some kind of identity contact and bond in real life. Second, the information of the WeChat circle of friends carries a kind of privacy. That is, only when both parties are friends, can they see each other's information. If both parties are friends with a third person, they can see the third person's comments. Under such a mechanism, the WeChat circle of friends is more like a virtual projection of a real circle of friends on the Internet. This mechanism determines that the WeChat circle of friends has certain image-building function. Information forwarded and noticed by one person can invisibly display a person's image and taste. Therefore, in a circle of friends, people tend to comment and forward with positive information. At the same time, it also led to the proliferation of "spirit chicken soup" content in the circle of friends.

Self-representation under the operating mechanism of Momo. Momo, as its name suggests, is marked by the strangers' social connection. Both the image and the information are anonymous. On such a platform, people can show their hidden inner desires and temporarily put down the shackles of their status. People can choose to take off some masks for a while and display them in everyday life. This kind of social media is more like a virtual projection of people's innermost privacy on the Internet. People's freedom is released at the most and their sense of responsibility is minimized. 
Under such a mechanism, people are self-represented by a person who is incomplete or a collection of certain desires. The platform has a relaxing function, but the value of information is low, even with a "yellow gambling" nature which is most likely to spread and spread.

\subsection{Self-presentation in social media is a projection of "realistic self"}

In the analysis above, social media naturally has a "mask" feature. Thus, it naturally possesses many virtual and deceptive features. However, in essence, self-presentation in social media still shows the "realistic self" of each individual person. In terms of projection on online media, in other words, the image that a person presents on the Internet is subject to their true abilities, hobbies and social status in life. To certain sense, social media focuses on a person's certain IQ and profile. Therefore, by paying close attention to the release of information of certain group of people on Microblog and WeChat, they can also roughly grasp the person's interests, hobbies and knowledge, as well as their key information, value propensity and other important information. Taking Microblog as an example, each person's reaction and commentary on the same incident often reflects his or her true values. For example, in the WeChat circle of friends, everyone will have their own friends encountered at different stages, including elementary school students, middle school students, high school students, undergraduates and masters. With long-term attention to the information and comments posted by the student circles at different stages, we can see that the influence of different educational levels on people will have relatively clear gradient in the micro-channel circle. In general, those with higher academic qualifications may send the information closed to their major. It is the sane with the tourist photos. Tourism information released by people of different living standards tends to have relatively clear "gradient" features.

\section{Some Suggestions on Guiding People to Realize "Positive Presentation" through System Adjustment}

As discussed in the previous section, from the macro perspective, people's online behavior still reflects the real society; from the micro perspective, people's behavior on the Internet shows a real individual. In other words, the way and content of people's "self-presentation" are not traceable. They are subject to various specific mechanisms and systems. Therefore, with the change of the media, there will be some disorder and confusion within certain period of time. However, we must be rational and inclusive. It must be realized that the network is essentially a medium and a tool. Therefore, disabling tools is a poorer response. The correct approach is to pass on their ideas through the Internet and guide people to achieve "positive presentation".

\subsection{Institutional adjustments should focus on embodying an "equalization" perspective}

Unlike traditional "top-down" advocacy methods, under the network environment, people have access to information that comes from all sides, showing some "flattening" characteristics. At the same time, subject to the concept of "popularization" of the Internet, netizens often have natural hostility to the official wording and methods. Therefore, in order to achieve the effect of publicity in the Internet environment, it is necessary to present an equalization. Only by abandoning the superior attitude, can we truly win the attention and support of netizens. For example, when the official media uses some official languages to promote ideas, these ideas are often ridiculed by netizens. If many policies and institutions can be presented in stories and comics, they will have a truly "popular" perspective and the ordinary people feel the impact on their own lives, this system can really play its due function.

\subsection{System adjustments should skillfully lead people to bring "positive energy" masks}

Through institutional analysis, we can clearly see that people choose and spread different content on different social media with different masks in order to achieve differentiated "self-presentation". The stronger the reality of communication behind social media, the easier it is to spread "positive energy". Therefore, according to the characteristics of different media platforms, different institutional adjustments are made to consciously guide authentic socialization, which is objectively 
conducive to guiding people to spread positive energy and achieve "positive" presentation in social media on the Internet. Based on this, implementing a real-name system for different media platforms must be effective to certain extent. And this real-name system is suitable for gradual promotion and implementation in a slow, gentle and covert manner.

\subsection{Institutional language should have the adaptability of network communication}

Limited by the time and the number of words, the information on the social media is presented with obvious "fragmentation" and "popularization" features. Therefore, when a system attempts to function through the Internet media, it must also adapt to the network environment. On one hand, it must be good at passing information through "fragmentation". On the other hand, such information must be with "popularization". For example, in the online media, there is a very special group of people called "Duanzishou" who are good at presenting information with certain ironic words.

In short, we must clearly understand that each of us can't live without a variety of masks. The "mask" itself has the essential meaning of "shading reality". But when one person puts on a mask for himself, it does not completely mean negative. Even to some extent, it will show a positive significance. At the same time, although "self-presentation in social media on the Internet" has many distinctive features and has had profound impact on individuals and society, what is still playing a role behind the Internet is still true in social and real life. Therefore, for online social media, we should maintain some kind of tolerance and acceptance attitude and guide people to achieve positive and effective "positive presentation" through system adjustment.

\section{References}

[1] Erving Goffman. The Presentation of Self in Everyday Life [M]. Peking University Press, 2014 (08).

[2] Z. Huang. Analysis on the "Self" in Goffman's Theory of Drama - Talking from the Book Named Self-rendering of Daily Life [J]. Journal of Shantou University (Humanities Edition). 2012 (12).

[3] W. Xiao. On Irving Goffman's Framework Thoughts [J]. Journal of International Communication. 2010 (12).

[4] Manning, P. Erving Goffman and modern sociology. 1992.

[5] J. J. Zhang. Analysis on Social Interaction in Virtual Social Network with Goffman's Theory of Drama Play [J]. Science of Social Psychology. 2015 (Z1).

[6] D. N. Luo, L. Luo. Self-representation in Mircoblog - Read Goffman's The Presentation of Self in Everyday Life [J]. Heihe Journal. 2014 (02).

[7] T. T. Wang. Self-rendering in social networks [J]. Theory Research. 2011 (17).

[8] Y. Huang. Perspective of Role Performing and Out-of-character Activities in Goffman's The Presentation of Self in Everyday Life [J]. Theory Research. 2013 (09).

[9] H. Ayihen. Goffman's Image Creation of Microblog Officials from the Perspective of "Foreground" Performance and "Backstage" Rendering [D]. Shaanxi Normal University. 2014 (05).

[10] L. F. Ruan. Daily Life and Literature in Shanghai - A Literature Review of "City as a Lifestyle" [D]. Shandong University. 2014 (10). 